\title{
Holographies and EXAFS in quantum electrodynamics
}

\author{
Y. Nishino* and G. Materlik \\ Hamburger Synchrotronstrahlungslabor HASYLAB at Deutsches Elektronen-Synchrotron DESY Notkestrasse 85, \\ D-22607 Hamburg, Germany
}

(Received 20 May 1999)

\begin{abstract}
We calculate the cross section of the photoionization process consistently in the framework of quantum electrodynamics, including the initial-state photon interaction and the final-state photoelectron interaction with neighboring atoms. The obtained photoionization cross section explains multiple energy x-ray holography $(\mathrm{MEXH})$, photoelectron holography, and extended x-ray absorption fine structure (EXAFS). In EXAFS, the contribution of the initial-state photon interaction is found to oscillate with energy and can disturb the EXAFS signal. It becomes non-negligible at higher energies. At $1 \mathrm{keV}$ above the absorption edge, it can be in comparable order of the EXAFS signal. The hologram functions of MEXH and x-ray fluorescence holography are consistent with those in classical electrodynamics. [S0163-1829(99)02546-1]
\end{abstract}

\section{INTRODUCTION}

Studies of the atomic structure of materials using $\mathrm{x}$ rays are of interest for a wide area of science. For crystalline materials, diffraction methods give well-established ways to study the lattice structure based on the long range order.

The extended x-ray absorption fine structure (EXAFS) method reveals the structure of neighboring atoms not only for crystalline materials but also for materials which only possess short range order. ${ }^{1}$ The EXAFS oscillations in the $\mathrm{X}$-ray absorption coefficient extend in the energy region 30-1500 eV above the absorption edge. They are explained by the interference of an outgoing photoelectron wave and the wave backscattered from neighboring atoms. An analysis of EXAFS gives the radial distribution of neighboring atoms.

A direct $\mathrm{x}$-ray optical image with atomic resolution has not been possible for various reasons. Towards this goal, Szöke has suggested to use Gabor's approach to realize atomic resolution holography with internal sources. ${ }^{2}$ Such holographic method can offer a promising way for structural studies. In the internal source holography, sources of electrons or photons are inside a sample, and a detector is situated at far distance. As internal sources, one can use, e.g., photoelectrons, ${ }^{3}$ diffusely scattered low energy electrons, ${ }^{4}$ Kikuchi electrons, ${ }^{5}$ Auger electrons, ${ }^{6}$ fluorescence photons, ${ }^{7}$ or bremsstrahlung photons. ${ }^{8}$ A particle wave from the source may directly reach the detector or may be coherently scattered by neighboring atoms before reaching the detector. These two waves serve as the reference wave and the object wave of holography, respectively, and form a holographic interference in the far field. By moving the detector position with respect to the sample, one measures a hologram. In order to obtain a three-dimensional atomic structure around source atoms from a hologram, a numerical reconstruction is used. ${ }^{3,9,10}$

In Ref. 11 another holographic method was proposed, in which the positions of detector and source are interchanged compared to the internal source holography. In the internal detector holography, photons ${ }^{11}$ or electrons ${ }^{12}$ are injected on a sample from an external source, and atoms inside the sample are used as detectors. The intensity of the incident particle wave at a position of an atom inside the sample changes according to the incident wave direction with respect to the sample. The change is caused by an interference of the unscattered incident wave and the wave coherently scattered by neighboring atoms, and can be interpreted as a hologram. In order to observe the wave intensity at the position of an atom, one measures, e.g., the fluorescence or the Auger electron yield from the atom, since they are proportional to the local wave intensity assuming that the energy of the incident wave is higher than the absorption edge of the atom.

In this paper we use the following terminology. The internal source holography with a fluorescence source is conventionally called $\mathrm{x}$-ray fluorescence holography (XFH), and the internal detector holography with incident $\mathrm{x}$ ray is referred to as multiple energy $\mathrm{x}$-ray holography (MEXH). Note, however, that this can be misleading. In the internal source holography with a fluorescence source, it is also possible to measure holograms at multiple energies by detecting different fluorescence lines. On the other hand, in the internal detector holography with incident photon, one may not always vary the incident photon energy as in the case using the Mössbauer source. ${ }^{13}$ Although there is a possible confusion in the terminology, we follow a conventional use of $\mathrm{XFH}$ and MEXH. We also use the widely accepted term of photoelectron holography $(\mathrm{PEH})$ as the internal source holography with a photoelectron source.

All the structure determination methods mentioned above have as a common feature that they make use of the interference of waves undergoing different paths. For example, in $\mathrm{MEXH}$ the interference of incident photons is used. And both PEH and EXAFS utilize the interference of photoelectrons.

If one looks at the problem from a different point of view, the photoionization process is involved in MEXH, PEH, and EXAFS. In the photoionization process, photons hit atoms, and emit photoelectrons. In this process, both the initial-state incident photon and the final-state photoelectron may interact with other atoms. In MEXH usually one takes into account the initial-state photon interaction, and not the final-state electron interaction. On the other hand, in PEH and EXAFS, 


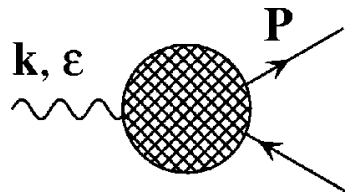

FIG. 1. The photoionization process. The incident photon produces a pair of photoelectron and core-hole state. In this figure, time passes from left to right. The wavy line is the incident photon with momentum $\boldsymbol{k}$, energy $\omega=|\boldsymbol{k}|$, and polarization $\boldsymbol{\epsilon}$. The straight line with the forward arrow in time is the photoelectron with momentum $\boldsymbol{p}$. The straight line with the backward arrow in time is the core-hole state. The circle with hatch represents all diagrams to be specified below.

it is common to neglect the initial-state photon interaction. Natural questions are how big are the effects of the final-state photoelectron interaction in MEXH and how big are the effects of the initial-state photon interaction in PEH and EXAFS. From the first sight, it seems to be plausible that the electron-atom interaction is much stronger than the photonatom interaction, and one can neglect the latter in PEH and EXAFS. Then how much is the ratio of these contributions? What does the latter contribution look like? Further questions remain. If the electron-atom interaction is so strong, how can one get holograms in MEXH? In this paper, we try to answer these questions by calculating the cross section of the photoionization process using quantum electrodynamics (QED), and including both the initial-state photon interaction and the final-state photoelectron interaction with neighboring atoms.

Concerning studies of these experimental methods in quantum theories, the EXAFS cross section has been intensely calculated. ${ }^{1}$ As for the holographic methods, few works were done in QED. ${ }^{8,14}$ Here we show explicit forms of the cross sections of MEXH and PEH in QED. Using a similar calculation technique, we also show the transition probability of XFH.

\section{DEFINITION OF CROSS SECTIONS}

We define the cross sections of MEXH, PEH, and EXAFS, and make a general discussion before deriving explicit expressions of them. Throughout this paper, we use the natural unit, $\hbar=c=\epsilon_{0}=1$, where $\hbar$ is the Plank constant divided by $2 \pi, c$ is the speed of light in vacuum, and $\epsilon_{0}$ is the permittivity of free space.

Let us first consider the photoionization process, which is the underlying physical process in all $\mathrm{MEXH}, \mathrm{PEH}$, and EXAFS. The photoionization process is schematically shown in Fig. 1. In the photoionization process, a pair of photoelectron and core-hole state is created by the incident photon. The excited core-hole state subsequently deexcites through the fluorescence process or the Auger process. The photoionization process is measured by detecting photoelectrons, fluorescence photons, Auger electrons, or loss of incident photons. The photoionization process can be considered as a separate quantum process from its deexcitation process, since the contribution of the intermediate core-hole state dominates in the probability amplitudes to find fluorescence photons and Auger electrons. The dominance of the core-hole state results in nearly isotropic distribution of fluorescence photons and Auger electrons.

For a free isolated atom, the photoionization cross section depends on the incident photon energy $\omega$ and the outgoing photoelectron direction $\hat{\boldsymbol{p}}$ relative to the incident photon direction $\hat{\boldsymbol{k}}$ and to the incident photon polarization $\boldsymbol{\epsilon}$. In the case that target atoms are spatially ordered, such as atoms in crystalline solids, the cross section depends independently on the outgoing photoelectron direction $\hat{\boldsymbol{p}}$, on the incident photon direction $\hat{\boldsymbol{k}}$, and on the incident photon polarization $\boldsymbol{\epsilon}$. The general form of the differential cross section of the photoionization process is thus given by

$$
\frac{d \sigma_{\mathrm{PI}}(\omega, \hat{\boldsymbol{k}}, \boldsymbol{\epsilon}, \hat{\boldsymbol{p}})}{d \Omega_{p}} .
$$

Here vectors with hats denote unit vectors with the same direction as vectors without hats, e.g., $\hat{\boldsymbol{k}}=\boldsymbol{k} /|\boldsymbol{k}|$. $\omega, \boldsymbol{k}$, and $\boldsymbol{\epsilon}$ are energy, momentum, and polarization vector of the incident photon, respectively. $\boldsymbol{p}$ and $\Omega_{\boldsymbol{p}}$ are momentum and solid angle of the outgoing photoelectron, respectively. All the cross sections of MEXH, PEH, and EXAFS are defined in terms of the photoionization cross section.

In MEXH, one observes the total cross section of the photoionization process while changing direction $\hat{\boldsymbol{k}}$ and energy $\omega$ of the incident photon. By changing the incident photon direction $\hat{\boldsymbol{k}}$, the incident photon polarization $\boldsymbol{\epsilon}$ also changes. The cross section of MEXH is given by

$$
\sigma_{\mathrm{MEXH}}(\omega, \hat{\boldsymbol{k}}, \boldsymbol{\epsilon})=\int_{4 \pi} d \Omega_{p} \frac{d \sigma_{\mathrm{PI}}(\omega, \hat{\boldsymbol{k}}, \boldsymbol{\epsilon}, \hat{\boldsymbol{p}})}{d \Omega_{p}}
$$

where $4 \pi$ at the integration symbol denotes that integration over solid angle runs over full $4 \pi$.

In $\mathrm{PEH}$, the outgoing photoelectron direction $\hat{\boldsymbol{p}}$ is measured for different incident photon energies $\omega$. Direction $\hat{\boldsymbol{k}}$ and polarization $\boldsymbol{\epsilon}$ of the incident photon are fixed at some values, e.g., $\hat{\boldsymbol{k}}=\hat{\boldsymbol{a}}$ and $\boldsymbol{\epsilon}=\boldsymbol{\epsilon}_{a}$. Therefore we have

$$
\sigma_{\mathrm{PEH}}(\omega, \hat{\boldsymbol{p}})=\frac{d \sigma_{\mathrm{PI}}\left(\omega, \hat{\boldsymbol{k}}=\hat{\boldsymbol{a}}, \boldsymbol{\epsilon}=\boldsymbol{\epsilon}_{a}, \hat{\boldsymbol{p}}\right)}{d \Omega_{p}} .
$$

In EXAFS, one measures the total cross section of the photoionization process as a function of the incident photon energy $\omega$. Direction $\hat{\boldsymbol{k}}$ and polarization $\boldsymbol{\epsilon}$ of the incident photon are fixed at some values, e.g., $\hat{\boldsymbol{k}}=\hat{\boldsymbol{a}}$ and $\boldsymbol{\epsilon}=\boldsymbol{\epsilon}_{a}$. Thus we obtain

$$
\sigma_{\mathrm{EXAFS}}(\omega)=\int_{4 \pi} d \Omega_{p} \frac{d \sigma_{\mathrm{PI}}\left(\omega, \hat{\boldsymbol{k}}=\hat{\boldsymbol{a}}, \boldsymbol{\epsilon}=\boldsymbol{\epsilon}_{a}, \hat{\boldsymbol{p}}\right)}{d \Omega_{p}}
$$

By comparing the cross sections shown above, one observes interesting relationships among them. From Eqs. (3) and (4), we get

$$
\sigma_{\mathrm{EXAFS}}(\omega)=\int_{4 \pi} d \Omega_{p} \sigma_{\mathrm{PEH}}(\omega, \hat{\boldsymbol{p}}) .
$$


Equation (5) means that EXAFS measurements are nothing but the photoelectron angular integral of PEH measurements. This reflects the fact that in EXAFS one gets only an averaged information about neighboring atoms. On the other hand in $\mathrm{PEH}$ one can, in principle, get three-dimensional information of neighboring atoms. In both PEH and EXAFS, the interference of final-state photoelectrons plays an essential role.

Another relationship is obtained by comparing Eqs. (2) and (4):

$$
\sigma_{\mathrm{EXAFS}}(\omega)=\sigma_{\mathrm{MEXH}}\left(\omega, \hat{\boldsymbol{k}}=\hat{\boldsymbol{a}}, \boldsymbol{\epsilon}=\boldsymbol{\epsilon}_{a}\right) .
$$

Equation (6) implies that EXAFS measurements correspond to MEXH measurements at an arbitrary incident photon direction $\hat{\boldsymbol{k}}$. Although in EXAFS experiments one does not measure the dependence of the cross section on the incident photon direction $\hat{\boldsymbol{k}}$, it also contains holographic information. In $\mathrm{MEXH}$, one is interested in the interference of incident photons.

At this point, one should be careful. In order to justify that the directional $\hat{\boldsymbol{k}}$ dependence of the MEXH cross section is caused merely by the incident photon, one has to assume that the initial state (before the incident photon reaches the atom which emits the photoelectron) is separated from the final state (after the photoelectron is emitted). But there is no good reason for justifying this assumption. One should describe the photoionization process in a single step picture. It is also clear from the experimental fact that the angle distribution of the outgoing photoelectron $\hat{\boldsymbol{p}}$ greatly depends on the direction of the incident photon polarization $\boldsymbol{\epsilon}$. Regarding the photoionization process as a single step process means that changes in the incident photon direction $\hat{\boldsymbol{k}}$ produce changes in the final state This results in another incident photon direction dependence of the cross section. In conventional treatments of $\mathrm{MEXH}$, it is implicitly assumed that the initial state of the photoionization process is a single step process, and the final-state photoelectrons are disregarded. On the other hand, in EXAFS and PEH, it is usual to disregard the effect of the photon interference in the initial state. By changing the incident photon energy $\omega$, the interference pattern of photons in the initial state also changes. In this paper, we consistently take into account both the initial-state interaction and the final-state interaction.

\section{CALCULATION OF CROSS SECTIONS}

\section{A. Cross section and transition matrix}

We calculate the photoionization cross section in QED. We assume that the kinematical region is where the incident photon energy is higher than the ionization energy of atoms, but smaller than the electron rest mass energy. We use this assumption to justify the nonrelativistic treatment of electrons, the plane wave approximation of photoelectrons, and the dominance of the Thomson scattering in the photon-atom interaction. Although this kinematical region is higher than what is of interest, it is good for the first approximation. In fact, the essential mechanism of EXAFS is explained within this assumption. ${ }^{1}$

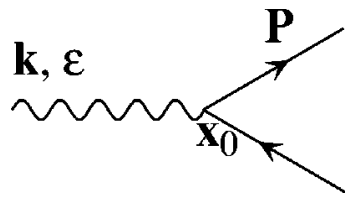

FIG. 2. The leading order contribution of the photoionization process. The incident photon scatters an electron bound in the atom at $\boldsymbol{X}_{0}$.

Since atoms are much heavier than electrons, we treat them as infinitely heavy objects without recoils. In this case, during the process, the total momentum does not conserve, while the total energy does. It is reasonable to define the transition matrix $T_{f i}$ as follows ${ }^{15}$ :

$$
S_{f i}=\delta_{f i}+2 \pi i \delta\left(E_{f}-E_{i}\right) T_{f i}
$$

where $S_{f i}$ is the $S$ matrix. $E_{f}$ and $E_{i}$ are the total energy of system in the final state and the initial state, respectively. The differential cross section of the photoionization process is defined in terms of the transition matrix

$$
\frac{d \sigma_{\mathrm{PI}}}{d \Omega_{p}}=\left|T_{f i}\right|^{2}(2 \pi)^{4}|\boldsymbol{p}| E,
$$

where $E$ is the energy of photoelectron $E=\sqrt{m_{\mathrm{e}}^{2}+|\boldsymbol{p}|^{2}} \approx m_{\mathrm{e}}$ $+\boldsymbol{p}^{2} / 2 m_{\mathrm{e}}$ and $m_{\mathrm{e}}$ is the mass of electron.

In the following, we show calculations of the photoionization transition matrix in detail including the phase factors which depend on positions of target atoms. Such phase factors are usually not considered in problems with a single target atom, since it becomes 1 by squaring the transition matrix. In the case of many target atoms sitting at different positions, however, the phase factors play essential roles to produce the interference of waves.

We calculate the transition matrix order by order in the perturbation series, but we neglect divergent contributions corresponding to, e.g., the self-interaction. These contributions should vanish after appropriate procedures of the renormalization.

\section{B. Leading order photoionization contribution}

The Feynman diagram of the leading order contribution of the photoionization process is given by Fig. 2. The $S$ matrix is given by

$$
S_{f i}^{(0)}=i e \int d^{3} r \int d t \quad \boldsymbol{J}^{F I}(\boldsymbol{r}, t) \cdot\langle 0|\boldsymbol{A}(\boldsymbol{r}, t)| \boldsymbol{k}, \boldsymbol{\epsilon}\rangle,
$$

where $e$ is the electron charge, $|0\rangle$ is the vacuum state of the photon (the zero-photon state), and $|\boldsymbol{k}, \boldsymbol{\epsilon}\rangle$ is the one-photon state with momentum $\boldsymbol{k}$ and polarization $\boldsymbol{\epsilon} \cdot \boldsymbol{A}(\boldsymbol{r}, t)$ is the photon field operator in the interaction representation. Note that for the photon field operator $\boldsymbol{A}(\boldsymbol{r}, t)$, we took the threedimensionally transverse gauge $A_{0}(\boldsymbol{r}, t)=0$, which is a convenient gauge fixing condition for nonrelativistic problems. ${ }^{15}$ $\boldsymbol{J}^{F I}(\boldsymbol{r}, t)$ in Eq. (9) is the electromagnetic current in the interaction representation, and for nonrelativistic particles it is given by 


$$
\begin{aligned}
\boldsymbol{J}^{F I}(\boldsymbol{r}, t)= & \frac{-i}{2 m_{\mathrm{e}}}\left[\psi_{F}^{*}(\boldsymbol{r}, t) \vec{\nabla} \psi_{I}\left(\boldsymbol{r}-\boldsymbol{X}_{0}, t\right)\right. \\
& \left.-\psi_{F}^{*}(\boldsymbol{r}, t) \overleftarrow{\nabla} \psi_{I}\left(\boldsymbol{r}-\boldsymbol{X}_{0}, t\right)\right],
\end{aligned}
$$

where $\psi_{F}(\boldsymbol{r}, t)$ is the final-state photoelectron wave function, $\psi_{I}\left(\boldsymbol{r}-\boldsymbol{X}_{0}, t\right)$ is the initial-state bound electron wave function, and $\boldsymbol{X}_{0}$ is the position of the photoelectron emitter atom. Note that if more than one initial atomic states contribute to the process, one should add all contributions.

Since integration over time $t$ in Eq. (9) gives the energy conservation delta function, we obtain the transition matrix of the leading order photoionization contribution

$$
T_{f i}^{(0)}=\frac{i}{m_{\mathrm{e}}} \frac{e}{\sqrt{(2 \pi)^{3} 2 \omega}} \int d^{3} r \psi_{F}^{*}(\boldsymbol{r}) \boldsymbol{\epsilon} \cdot \overleftarrow{\nabla} \psi_{I}\left(\boldsymbol{r}-\boldsymbol{X}_{0}\right) e^{i \boldsymbol{k} \cdot \boldsymbol{r}} .
$$

If the incident photon energy $\omega$ is higher than the ionization energy, the Born approximation is applicable. Thus we take the final-state photoelectron as plane wave:

$$
\psi_{F}(\boldsymbol{r})=\frac{1}{\sqrt{(2 \pi)^{3}}} e^{i \boldsymbol{p} \cdot \boldsymbol{r}}
$$

In this case, the transition matrix becomes

$$
T_{f i}^{(0)}=C(\omega, \hat{\boldsymbol{p}} \cdot \hat{\boldsymbol{k}}) e^{-i(\boldsymbol{p}-\boldsymbol{k}) \cdot \boldsymbol{X}_{0}} \boldsymbol{\epsilon} \cdot \hat{\boldsymbol{p}}
$$

with

$$
C(\omega, \hat{\boldsymbol{p}} \cdot \hat{\boldsymbol{k}})=\frac{e \beta}{(2 \pi)^{3} \sqrt{2 \omega}} \int d^{3} s_{0} \psi_{I}\left(\boldsymbol{s}_{0}\right) e^{-i(\boldsymbol{p}-\boldsymbol{K}) \cdot s_{0}} .
$$

Here we changed integration variables from $\boldsymbol{r}$ to $\boldsymbol{s}_{0}$ according to $\boldsymbol{r}=\boldsymbol{X}_{0}+\boldsymbol{s}_{0} \cdot \beta=|\boldsymbol{p}| / m_{\mathrm{e}}$ is the velocity of the photoelectron.

As an example, we show an explicit expression of $C(\omega, \hat{\boldsymbol{p}} \cdot \hat{\boldsymbol{k}})$ in the case where the initial state is the hydrogenlike atom:

$$
\psi_{I}\left(s_{0}\right)=\left(\frac{Z^{3}}{\pi a_{0}^{3}}\right)^{1 / 2} e^{-Z\left|s_{0}\right| / a_{0}}
$$

where $Z$ is the atomic number, $a_{0}$ is the Bohr radius $a_{0}$ $=1 / m_{\mathrm{e}} \alpha$, and $\alpha$ is the fine-structure constant $\alpha=e^{2} / 4 \pi$ $\approx 1 / 137$. As one can easily find in textbooks on quantum mechanics, ${ }^{16}$ the coefficient $C(\omega, \hat{\boldsymbol{p}} \cdot \hat{\boldsymbol{k}})$ in this case is given by

$$
C(\omega, \hat{\boldsymbol{p}} \cdot \hat{\boldsymbol{k}})=\frac{e}{4 \pi^{5 / 2} \omega^{2}}(Z \alpha)^{5 / 2} \frac{1}{(1-\beta \hat{\boldsymbol{p}} \cdot \hat{\boldsymbol{k}})^{2}} .
$$

Here we have used the fact that the ionization energy, $I$ $=\frac{1}{2}(z \alpha)^{2} m_{\mathrm{e}}$ is much smaller than the photon energy $\omega$. It should be noted that $C(\omega, \hat{\boldsymbol{p}} \cdot \hat{\boldsymbol{k}})$ is almost independent of $\hat{\boldsymbol{p}} \cdot \hat{\boldsymbol{k}}$, since the velocity $\beta$ of photoelectron is small. In the following we regard $C(\omega, \hat{\boldsymbol{p}} \cdot \hat{\boldsymbol{k}})$ as angle independent $C(\omega, \hat{\boldsymbol{p}} \cdot \hat{\boldsymbol{k}}) \rightarrow C(\omega)$.

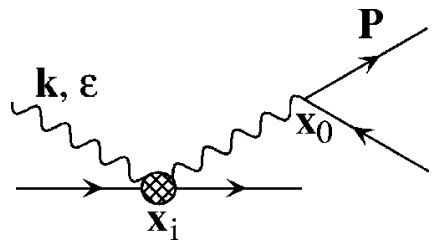

FIG. 3. The scattering of the photon in the initial state. The photon is scattered by an electron of the atom at $\boldsymbol{X}_{i}$. In QED, the virtual photon travels in both directions: from the atom at $\boldsymbol{X}_{i}$ to the one at $\boldsymbol{X}_{0}$ and from the atom at $\boldsymbol{X}_{0}$ to the one at $\boldsymbol{X}_{i}$. The circle with hatch contains all photon-electron interaction diagrams. For the nonrelativistic electron, they are the uncrossed, the crossed and the seagull diagrams. Among them, the Thomson scattering corresponds to the seagull diagram.

\section{Initial-state photon interaction}

The Feynman diagrams of the next-to-leading order contributions of the photoionization process are given by Figs. 3 and 4, where the initial-state photon and the final-state photoelectron interact with atoms, respectively.

Let us first consider photon interactions in the initial state. This contribution is composed of the photon-electron scattering and the leading order photoionization process. The cross section of the nonrelativistic photon-electron scattering is, in general, given by the Kramers-Kronig relation. The Kramers-Kronig relation explains both the Rayleigh scattering and the Thomson scattering. In the case where the incident photon energy is much higher than the ionization energy of atoms, the Thomson scattering dominates. The $S$ matrix of the Thomson scattering is given by

$$
\begin{aligned}
S^{(\text {Thomson })}= & -i \frac{e^{2}}{2 m_{\mathrm{e}}} \int d^{3} r \int d t\left\langle\boldsymbol{k}^{\prime}, \boldsymbol{\epsilon}^{\prime}|\boldsymbol{A}(\boldsymbol{r}, t) \cdot \boldsymbol{A}(\boldsymbol{r}, t)| \boldsymbol{k}, \boldsymbol{\epsilon}\right\rangle \\
& \times \sum_{i} \rho_{i}^{\mathrm{e}}\left(\boldsymbol{r}-\boldsymbol{X}_{i}\right)
\end{aligned}
$$

where $\boldsymbol{k}$ and $\boldsymbol{\epsilon}$ are momentum and polarization vector of the incident photon, respectively; $\boldsymbol{k}^{\prime}$ and $\boldsymbol{\epsilon}^{\prime}$ are momentum and polarization vector of the outgoing photon, respectively; $\rho_{i}^{e}\left(\boldsymbol{r}-\boldsymbol{X}_{i}\right)$ is the electron probability density of the $i$ th atom; and $\boldsymbol{X}_{i}$ is the position of the $i$ th atom. The sum over $i$ runs over all atoms in system. After integration over time $t$, we have the Thomson scattering transition matrix

$$
T^{\text {(Thomson })}=-r_{\mathrm{e}} \frac{1}{(2 \pi)^{2} \omega} \boldsymbol{\epsilon} \cdot \boldsymbol{\epsilon}^{\prime} \sum_{i} f_{i}^{\mathrm{x}}\left(\left|\boldsymbol{k}^{\prime}-\boldsymbol{k}\right|\right) e^{-i\left(\boldsymbol{k}^{\prime}-\boldsymbol{k}\right) \cdot \boldsymbol{X}_{i}},
$$

where $r_{\mathrm{e}}$ is the classical electron radius: $r_{\mathrm{e}}=\alpha / m_{\mathrm{e}}$. The form factor $f_{i}^{\mathrm{x}}$ of the photon scattering by the $i$ th atom is given by

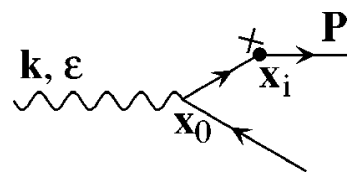

FIG. 4. The scattering of the photoelectron in the final state. The photoelectron is scattered by the Coulomb field of the atom at $\boldsymbol{X}_{i}$. 


$$
f_{i}^{\mathrm{X}}\left(\left|\boldsymbol{k}^{\prime}-\boldsymbol{k}\right|\right)=\int d s_{i}^{3} \rho_{i}^{\mathrm{e}}\left(\boldsymbol{s}_{i}\right) e^{-i\left(\boldsymbol{k}^{\prime}-\boldsymbol{k}\right) \cdot s_{i}},
$$

where $\boldsymbol{s}_{i}$ is the position vector measured from the center of the $i$ th atom $\boldsymbol{X}_{i}: \boldsymbol{s}_{i}=\boldsymbol{r}-\boldsymbol{X}_{i}$.

The $S$ matrix corresponding to Fig. 3 is given by

$$
\begin{aligned}
S_{f i}^{(1)}= & \frac{e^{3}}{m_{\mathrm{e}}} \sum_{m, n=1}^{3} \int d^{3} r_{1} \int d t_{1} \int d^{3} r_{2} \int d t_{2} J_{m}^{F I}\left(\boldsymbol{r}_{1}, t_{1}\right) \\
& \times\left\langle 0\left|T\left[A_{m}\left(\boldsymbol{r}_{1}, t_{1}\right), A_{n}\left(\boldsymbol{r}_{2}, t_{2}\right)\right]\right| 0\right\rangle \\
& \times\left\langle 0\left|A_{n}\left(\boldsymbol{r}_{2}, t_{2}\right)\right| \boldsymbol{k}, \boldsymbol{\epsilon}\right\rangle \sum_{i} \rho_{i}^{\mathrm{e}}\left(\boldsymbol{r}_{2}-\boldsymbol{X}_{i}\right),
\end{aligned}
$$

where variables with the suffix $m$ (or $n$ ) denote the $m$ th (or $n$ th) component of vectors.

For the following calculations, it is useful to define the photon propagator $G_{m n}\left(\omega^{\prime}, \boldsymbol{r}_{1}-\boldsymbol{r}_{2}\right)$ in the mixed frequencycoordinate representation in terms of the time-ordered product of the photon field operators

$$
\begin{aligned}
\langle 0| T & {\left[A_{m}\left(\boldsymbol{r}_{1}, t_{1}\right), A_{n}\left(\boldsymbol{r}_{2}, t_{2}\right)\right]|0\rangle } \\
& =-i \int \frac{d \omega^{\prime}}{2 \pi} e^{-i \omega^{\prime}\left(t_{1}-t_{2}\right)} G_{m n}\left(\omega^{\prime}, \boldsymbol{r}_{1}-\boldsymbol{r}_{2}\right) .
\end{aligned}
$$

Note that in the photon propagator, which corresponds to the virtual photon between an electron belonging to the atom at $\boldsymbol{X}_{i}$ and one belonging to the atom at $\boldsymbol{X}_{0}$, we have taken into account photons traveling in both directions (from the atom at $\boldsymbol{X}_{i}$ to the one at $\boldsymbol{X}_{0}$, and from the atom at $\boldsymbol{X}_{0}$ to the one at $\boldsymbol{X}_{i}$ ) and with all possible momenta and polarizations. Here the virtual photon means the photon appearing in quantum intermediate states. It is a pure quantum object and may break the on-shell condition due to the uncertainty principle of quantum theory: For the virtual photon $\omega \neq|\boldsymbol{k}|$.

In the three-dimensional transverse gauge, the photon propagator $G_{m n}(\omega, \boldsymbol{r})$ in the mixed frequency coordinate representation is given by ${ }^{15,17}$

$$
\begin{aligned}
G_{m n}(\omega, \boldsymbol{r})= & -\int \frac{d^{3} k}{(2 \pi)^{3}} \frac{e^{i \boldsymbol{k} \cdot \boldsymbol{r}}}{\omega^{2}-|\boldsymbol{k}|^{2}+i \epsilon}\left(\delta_{m n}-\frac{k_{m} k_{n}}{\omega^{2}}\right) \\
= & \frac{e^{i \omega r}}{4 \pi r}\left[\delta_{m n}\left(1+\frac{i}{\omega r}-\frac{1}{(\omega r)^{2}}\right)\right. \\
& \left.-\hat{r}_{m} \hat{r}_{n}\left(1+\frac{3 i}{\omega r}-\frac{3}{(\omega r)^{2}}\right)\right] .
\end{aligned}
$$

With the help of Eq. (21), integrations over $t_{1}, t_{2}$, and $\omega$ of the $S$ matrix Eq. (20) are carried out, and we have the transition matrix $T_{f i}^{(1)}$ :

$$
\begin{aligned}
T_{f i}^{(1)}= & -\frac{e^{3}}{m_{\mathrm{e}}} \sum_{m, n=1}^{3} \sum_{i} \int d^{3} r_{1} \int d^{3} r_{2} \frac{p_{m} \boldsymbol{\epsilon}_{n}}{(2 \pi)^{3} \sqrt{2 \omega}} \\
& \times e^{-i \boldsymbol{p} \cdot \boldsymbol{r}_{1}} \psi_{i}\left(\boldsymbol{r}_{1}-\boldsymbol{X}_{0}\right) G_{m n}\left(|\boldsymbol{k}|, \boldsymbol{r}_{1}-\boldsymbol{r}_{2}\right) e^{i \boldsymbol{k} \cdot \boldsymbol{r}_{2}} \rho_{i}^{\mathrm{e}}\left(\boldsymbol{r}_{2}-\boldsymbol{X}_{i}\right) .
\end{aligned}
$$

In order to perform integrations in Eq. (23), we make use of the fact that electrons are locally concentrated around the atomic positions and interatomic distances are much larger than the scale of atoms. ${ }^{1,8}$ In other words, the atomic wave function $\psi_{I}\left(s_{0}\right)$ and the electron probability density $\rho_{i}^{\mathrm{e}}\left(\boldsymbol{s}_{i}\right)$ have nonzero values only in the region $\left|s_{0}\right|,\left|s_{i}\right| \ll 1 /|\boldsymbol{k}|$ and the interatomic distance $\left|\boldsymbol{X}_{i}-\boldsymbol{X}_{0}\right|$ is much larger than this region of $\left|s_{0}\right|$ and $\left|s_{i}\right|$. Here the vectors $s_{0}$ and $s_{i}$ are the position vectors from the center of the zeroth atom and the $i$ th atom, respectively: $\boldsymbol{r}_{1}=\boldsymbol{X}_{0}+\boldsymbol{s}_{0}$ and $\boldsymbol{r}_{i}=\boldsymbol{X}_{i}+\boldsymbol{s}_{i}$. We also assume that the wavelength of the incident photon is smaller than the inter-atomic distance $\left|\boldsymbol{X}_{i}-\boldsymbol{X}_{0}\right|$. With the above consideration in mind, we make the following approximation:

$$
\begin{aligned}
G_{m n}\left(|\boldsymbol{k}|, \boldsymbol{r}_{1}-\boldsymbol{r}_{2}\right) \rightarrow & \frac{1}{4 \pi\left|\boldsymbol{\xi}_{i}\right|} e^{i|\boldsymbol{k}| \boldsymbol{\xi}_{i} \mid} e^{i|\boldsymbol{k}| \hat{\xi}_{i} \cdot\left(s_{i}-s_{0}\right)} \\
& \times\left[\delta_{m n}-\left(\hat{\xi}_{i}\right)_{m}\left(\hat{\xi}_{i}\right)_{n}\right],
\end{aligned}
$$

where $\boldsymbol{\xi}_{i}=\boldsymbol{X}_{i}-\boldsymbol{X}_{0}$. In Eq. (24), we used $\mid\left(\boldsymbol{X}_{i}+\boldsymbol{s}_{i}\right)-\left(\boldsymbol{X}_{0}\right.$ $\left.+\boldsymbol{s}_{0}\right)|\approx| \boldsymbol{\xi}_{i} \mid+\hat{\boldsymbol{\xi}}_{i} \cdot\left(\boldsymbol{s}_{i}-\boldsymbol{s}_{0}\right)$ in the phase factor and $\mid\left(\boldsymbol{X}_{i}+\boldsymbol{s}_{i}\right)$ $-\left(\boldsymbol{X}_{0}+\boldsymbol{s}_{0}\right)|\approx| \boldsymbol{\xi}_{i} \mid$ in other places; and we neglect higher order terms in $1 /|\boldsymbol{k}|\left|\boldsymbol{\xi}_{i}\right|$.

With the approximation in Eq. (24), integrations on $s_{i}$ and $s_{0}$ in Eq. (23) separate, and we finally obtain the transition matrix corresponding to Fig. 3:

$$
\begin{aligned}
T_{f i}^{(1)}= & -C(\omega) e^{-i(\boldsymbol{p}-\boldsymbol{k}) \cdot \boldsymbol{X}_{0}} r_{\mathrm{e}} \\
& \times \sum_{i \neq 0}\left(\boldsymbol{\epsilon} \cdot \hat{\boldsymbol{p}}-\boldsymbol{\epsilon} \cdot \hat{\boldsymbol{\xi}}_{i} \hat{\boldsymbol{p}} \cdot \hat{\boldsymbol{\xi}}_{i}\right) \frac{f_{i}^{\mathrm{X}}\left(\left|\boldsymbol{l}_{i}\right|\right)}{\left|\boldsymbol{\xi}_{i}\right|} e^{i|\boldsymbol{k}| \boldsymbol{\xi}_{i} \mid} e^{i \boldsymbol{k} \cdot \boldsymbol{\xi}_{i}},
\end{aligned}
$$

where $\boldsymbol{l}_{i}$ is the momentum transfer at the Thomson scattering: $\boldsymbol{l}_{i}=-|\boldsymbol{k}| \hat{\boldsymbol{\xi}}_{i}-\boldsymbol{k}$. Note that we have excluded the term $i$ $=0$ in the summation of Eq. (25). It corresponds to the selfinteraction of atoms with photons, and is divergent. As we mentioned before, divergent terms should vanish after appropriate procedures of the renormalization.

\section{Final-state photoelectron interaction}

The final-state photoelectron contribution, corresponding to Fig. 4, is composed of the Coulomb scattering and the leading order photoionization process. The transition matrix of the Coulomb scattering by the $i$ th atom is given by

$$
S_{i}^{(\text {Coulomb })}=-i e \int d^{3} r \int d t \sum_{i} \phi_{i}\left(\boldsymbol{r}-\boldsymbol{X}_{i}\right) J_{0}^{p p^{\prime}}(\boldsymbol{r}),
$$

where $\boldsymbol{p}^{\prime}$ is the initial-state electron momentum and $\boldsymbol{p}$ is the final-state electron momentum. $\phi_{i}\left(\boldsymbol{r}-\boldsymbol{X}_{i}\right)$ is the classical Coulomb potential of the $i$ th atom

$$
\phi_{i}\left(s_{i}\right)=\frac{-e}{4 \pi} \int d^{3} s_{i}^{\prime} \frac{\rho_{i}\left(s_{i}^{\prime}\right)}{\left|s_{i}-s_{i}^{\prime}\right|},
$$

and $\rho_{i}\left(\boldsymbol{s}_{i}\right)$ is the charge density of the $i$ th atom. $J_{0}^{p p^{\prime}}(\boldsymbol{r})$ is the zeroth component of the electron electromagnetic current. In the nonrelativistic case, it is given by

$$
J_{0}^{p p^{\prime}}(\boldsymbol{r})=\psi_{p}^{*}(\boldsymbol{r}) \psi_{p^{\prime}}(\boldsymbol{r}),
$$


where $\psi_{p^{\prime}}(\boldsymbol{r})$ and $\psi_{p}(\boldsymbol{r})$ are wave functions of electrons before and after the Coulomb scattering, respectively.

In the Born approximation, the transition matrix of the Coulomb scattering is given by

$$
\begin{aligned}
T^{(\text {Coulomb })} & =\frac{-e}{(2 \pi)^{3}} \sum_{i} \int d^{3} r \phi_{i}\left(\boldsymbol{r}-\boldsymbol{X}_{i}\right) e^{-i\left(\boldsymbol{p}-\boldsymbol{p}^{\prime}\right) \cdot \boldsymbol{r}} \\
& =\sum_{i} T_{i}^{(\text {Coulomb })} e^{-i\left(\boldsymbol{p}-\boldsymbol{p}^{\prime}\right) \cdot \boldsymbol{X}_{i},}
\end{aligned}
$$

where

$$
T_{i}^{(\text {Coulomb })}=\frac{-e}{(2 \pi)^{3}} \int d^{3} s_{i} \phi_{i}\left(s_{i}\right) e^{-i\left(\boldsymbol{p}-\boldsymbol{p}^{\prime}\right) \cdot s_{i}} .
$$

The scattering amplitude $f_{i}^{\mathrm{e}}$ of the electron-atom scattering is defined in terms of the Coulomb scattering transition amplitude $T_{i}^{(\text {Coulomb) }}$, Eq. (30), as

$$
f_{i}^{\mathrm{e}}\left(\left|\boldsymbol{p}-\boldsymbol{p}^{\prime}\right|\right)=(2 \pi)^{2} m_{\mathrm{e}} T_{i}^{(\text {Coulomb })} .
$$

In the Mott-Bethe formula, it is related to the form factor $f_{i}^{\mathrm{X}}$, Eq. (19), of the photon-atom scattering: ${ }^{16,18}$

$$
f_{i}^{\mathrm{e}}(|\boldsymbol{q}|)=\frac{2 m_{\mathrm{e}} \alpha}{|\boldsymbol{q}|^{2}}\left[Z_{i}-f_{i}^{\mathrm{x}}(|\boldsymbol{q}|)\right],
$$

where $Z_{i}$ is the atomic number of the $i$ th atom. The first term is the contribution of the nucleus and the second term is that of electrons.

The calculation of the transition matrix of the final-state photoelectron interaction is done in a similar way as in Sec. III C. Details of calculations are also found in papers on theoretical treatments of EXAFS. ${ }^{1}$ For completeness of our discussion, we show the outline of calculations. The $S$ matrix corresponding to Fig. 4 is given by

$$
\begin{aligned}
S_{f i}^{(2)}= & e^{2} \int d^{3} r_{1} \int d t_{1} \int d^{3} r_{2} \int^{t_{1}} d t_{2} \int d^{3} p^{\prime} \\
& \times \sum_{i} \phi_{i}\left(\boldsymbol{r}-\boldsymbol{X}_{i}\right) J_{0}^{p p^{\prime}}\left(\boldsymbol{r}_{1}, t_{1}\right) \\
& \times \boldsymbol{J}^{p^{\prime} I}\left(\boldsymbol{r}_{2}, t_{2}\right) \cdot\left\langle 0\left|\boldsymbol{A}\left(\boldsymbol{r}_{2}, t_{2}\right)\right| \boldsymbol{k}, \boldsymbol{\epsilon}\right\rangle .
\end{aligned}
$$

After integrations over $t_{1}$ and $t_{2}$, we have the transition ma$\operatorname{trix} T_{f i}^{(2)}$ :

$$
\begin{aligned}
T_{f i}^{(2)}= & e^{2} \sum_{i} \int d^{3} r_{1} \int d^{3} r_{2} \int d^{3} p^{\prime} \\
& \times \frac{1}{\omega-\left(\left|\boldsymbol{p}^{\prime}\right|^{2} / 2 m_{\mathrm{e}}\right)-I+i \boldsymbol{\epsilon}} \boldsymbol{\phi}_{i}\left(\boldsymbol{r}-\boldsymbol{X}_{i}\right) \\
& \times J_{0}^{p p^{\prime}}\left(\boldsymbol{r}_{1}, t_{1}\right) \boldsymbol{J}^{p^{\prime} I}\left(\boldsymbol{r}_{2}, t_{2}\right) \cdot\left\langle 0\left|\boldsymbol{A}\left(\boldsymbol{r}_{2}, t_{2}\right)\right| \boldsymbol{k}, \boldsymbol{\epsilon}\right\rangle \\
= & -\frac{e^{2}}{(2 \pi)^{4} \sqrt{2 \omega}} \sum_{i} \int d^{3} r_{1} \int d^{3} r_{2} \frac{e^{i|\boldsymbol{p}|\left|\boldsymbol{r}_{1}-\boldsymbol{r}_{2}\right|}}{\left|\boldsymbol{r}_{1}-\boldsymbol{r}_{2}\right|} \\
& \times \phi_{i}\left(\boldsymbol{r}-\boldsymbol{X}_{i}\right) e^{-i \boldsymbol{p} \cdot \boldsymbol{r}_{1}\left(-i \vec{\nabla}_{2} \cdot \boldsymbol{\epsilon}\right) \psi_{I}\left(\boldsymbol{r}_{2}-\boldsymbol{X}_{0}\right) e^{i \boldsymbol{k} \cdot \boldsymbol{r}_{2}},}
\end{aligned}
$$

where $I$ is the ionization energy of the photoelectron emitter atom. In order to perform integrations over $\boldsymbol{r}_{1}$ and $\boldsymbol{r}_{2}$, we make use of a similar approximation as Eq. (24):

$$
\frac{e^{i|\boldsymbol{p}|\left|\boldsymbol{r}_{1}-\boldsymbol{r}_{2}\right|}}{\left|\boldsymbol{r}_{1}-\boldsymbol{r}_{2}\right|} \rightarrow \frac{1}{\left|\boldsymbol{\xi}_{i}\right|} e^{i|\boldsymbol{p}|\left|\boldsymbol{\xi}_{i}\right|} e^{i|\boldsymbol{p}| \hat{\boldsymbol{\xi}}_{i} \cdot\left(s_{i}-\boldsymbol{s}_{0}\right)}
$$

With the approximation Eq. (35), we integrate over $r_{1}$ and $r_{2}$ in Eq. (34), and finally obtain the expression of the transition matrix corresponding to Fig. 4:

$$
T_{f i}^{(2)}=C(\omega) e^{-i(\boldsymbol{p}-\boldsymbol{k}) \cdot \boldsymbol{x}_{0}} \sum_{i \neq 0} \boldsymbol{\epsilon} \cdot \hat{\boldsymbol{\xi}}_{i} \frac{f_{i}^{\mathrm{e}}\left(\left|\boldsymbol{q}_{i}\right|\right)}{\left|\boldsymbol{\xi}_{i}\right|} e^{i\left|\boldsymbol{p} \| \boldsymbol{\xi}_{i}\right|} e^{-i \boldsymbol{p} \cdot \boldsymbol{\xi}_{i}},
$$

where $\boldsymbol{q}_{i}$ is the momentum transfer at the Coulomb scattering: $\boldsymbol{q}_{i}=\boldsymbol{p}-|\boldsymbol{p}| \hat{\boldsymbol{\xi}}_{i}$. We have excluded the term $i=0$ in the summation of Eq. (36), which corresponds to the selfinteraction of atoms with the electrons.

\section{E. Cross section of photoionization process}

By summing Eqs. (13), (25), and (36), we obtain

$$
\begin{aligned}
T_{f i}= & C(\omega) e^{-i(\boldsymbol{p}-\boldsymbol{k}) \cdot \boldsymbol{X}_{0}} \\
& \times\left[\boldsymbol{\epsilon} \cdot \hat{\boldsymbol{p}}-r_{\mathrm{e}} \sum_{i \neq 0}\left(\boldsymbol{\epsilon} \cdot \hat{\boldsymbol{p}}-\boldsymbol{\epsilon} \cdot \hat{\boldsymbol{\xi}} \hat{\boldsymbol{p}} \cdot \hat{\boldsymbol{\xi}}_{i}\right) \frac{f_{i}^{\mathrm{x}}\left(\left|\boldsymbol{l}_{i}\right|\right)}{\left|\boldsymbol{\xi}_{i}\right|} e^{i|\boldsymbol{k}|\left|\boldsymbol{\xi}_{i}\right|} e^{i \boldsymbol{k} \cdot \boldsymbol{\xi}_{i}}\right. \\
& \left.+\sum_{i \neq 0} \boldsymbol{\epsilon} \cdot \hat{\boldsymbol{\xi}}_{i} \frac{f_{i}^{\mathrm{e}}\left(\left|\boldsymbol{q}_{i}\right|\right)}{\left|\boldsymbol{\xi}_{i}\right|} e^{i\left|\boldsymbol{p} \| \boldsymbol{\xi}_{i}\right|} e^{-i \boldsymbol{p} \cdot \boldsymbol{\xi}_{i}}\right]
\end{aligned}
$$

We again note that $\boldsymbol{\xi}_{i}$ is a vector from the atom at $\boldsymbol{X}_{0}$, which emits a photoelectron, to another atom at $\boldsymbol{X}_{i}: \boldsymbol{\xi}_{i}=\boldsymbol{X}_{i}-\boldsymbol{X}_{0}$.

By substituting the expression of the photoionization transition matrix Eq. (37) into the definition of the cross section Eq. (8), we obtain the cross section of the photoionization process:

$$
\begin{aligned}
\frac{d \sigma_{\mathrm{PI}}(\omega, \hat{\boldsymbol{k}}, \boldsymbol{\epsilon}, \hat{\boldsymbol{p}})}{d \Omega \boldsymbol{p}}= & (2 \pi)^{4} m_{\mathrm{e}}|\boldsymbol{p} \| C(\omega)|^{2}\left[(\boldsymbol{\epsilon} \cdot \hat{\boldsymbol{p}})^{2}-\boldsymbol{\epsilon} \cdot \hat{\boldsymbol{p}} \chi^{\mathrm{x}}(\omega, \hat{\boldsymbol{k}})\right. \\
& \left.+\boldsymbol{\epsilon} \cdot \hat{\boldsymbol{p}} \chi^{\mathrm{e}}(\omega, \hat{\boldsymbol{p}}, \boldsymbol{\epsilon})+\chi_{2}^{\mathrm{e}}(\omega, \hat{\boldsymbol{p}}, \boldsymbol{\epsilon})\right]
\end{aligned}
$$

where

$$
\begin{gathered}
\chi^{\mathrm{x}}(\omega, \hat{\boldsymbol{k}})=2 r_{\mathrm{e}} \operatorname{Re} \sum_{i \neq 0}\left(\boldsymbol{\epsilon} \cdot \hat{\boldsymbol{p}}-\boldsymbol{\epsilon} \cdot \hat{\boldsymbol{\xi}} \hat{\boldsymbol{p}} \cdot \hat{\boldsymbol{\xi}}_{i}\right) \frac{f_{i}^{\mathrm{x}}\left(\left|\boldsymbol{l}_{i}\right|\right)}{\left|\boldsymbol{\xi}_{i}\right|} e^{i|\boldsymbol{k}|\left|\boldsymbol{\xi}_{i}\right|} e^{i \boldsymbol{k} \cdot \boldsymbol{\xi}_{i}} \\
\chi^{\mathrm{e}}(\omega, \hat{\boldsymbol{p}}, \boldsymbol{\epsilon})=2 \operatorname{Re} \sum_{i \neq 0} \boldsymbol{\epsilon} \cdot \hat{\boldsymbol{\xi}}_{i} \frac{f_{i}^{\mathrm{e}}\left(\left|\boldsymbol{q}_{i}\right|\right)}{\left|\boldsymbol{\xi}_{i}\right|} e^{i|\boldsymbol{p}|\left|\boldsymbol{\xi}_{i}\right|} e^{-i \boldsymbol{p} \cdot \boldsymbol{\xi}_{i}}
\end{gathered}
$$

and

$$
\chi_{2}^{\mathrm{e}}(\omega, \hat{\boldsymbol{p}}, \boldsymbol{\epsilon})=\left|\sum_{i \neq 0} \boldsymbol{\epsilon} \cdot \hat{\boldsymbol{\xi}}_{i} \frac{f_{i}^{\mathrm{e}}\left(\left|\boldsymbol{q}_{i}\right|\right)}{\left|\boldsymbol{\xi}_{i}\right|} e^{i|\boldsymbol{p}|\left|\boldsymbol{\xi}_{i}\right|} e^{-i \boldsymbol{p} \cdot \boldsymbol{\xi}_{i}}\right|^{2} .
$$

In Eq. (38), we have neglected terms which are small and irrelevant to the following discussions. The cross section Eq. 
(38) of the photoionization process is composed of the direct term (the first term), the incident $\mathrm{x}$-ray interference (XI) term (the second term), the photoelectron hologram (PEH) term (the third term), and the electron self-interference (ESI) term (the fourth term).

\section{CROSS SECTION OF MEXH, PEH, AND EXAFS}

\section{A. Cross section of PEH}

From the definition, Eq. (3), the PEH cross section is given by the photoionization cross section Eq. (38) with fixed incident photon direction and polarization. Let us estimate the relative size of each term in the cross section Eq. (38). We show maximum contributions (the case of almost forward scattering in the Thomson scattering and the Coulomb scattering) of one neighboring atom sitting at distance $r$ from the photoelectron emitter atom. The approximate ratio is

direct term:XI term:PEH term:ESI term

$$
\approx 1: \frac{Z r_{e}}{r}: \frac{Z \alpha^{2}\left\langle r^{2}\right\rangle}{3 r_{\mathrm{e}} r}:\left(\frac{Z \alpha^{2}\left\langle r^{2}\right\rangle}{3 r_{\mathrm{e}} r}\right)^{2},
$$

where $\left\langle r^{2}\right\rangle$ and $Z$ are the mean square radius and the atomic number of the neighboring atom, respectively. In Eq. (42), we used the formula $f_{i}^{\mathrm{e}}(0)=Z \alpha^{2}\left\langle r^{2}\right\rangle / 3 r_{\mathrm{e}}$, which is obtained from the Mott-Bethe formula Eq. (32).

For medium heavy atoms ( $Z$ is in the order of 10) at $r$ $\approx$ (a few $\AA$ ), the direct term, the PEH term, and the ESI term are in comparable order. For the estimation of the PEH term and the ESI term, we referred to the tabulated values in Ref. [18] of the electron-atom scattering amplitudes $f_{i}^{\mathrm{e}}$ at almost forward direction. The XI term is much smaller by order $10^{-4}$ compared to the other terms. Thus one can neglect the effect of the initial-state photon interaction in the photoelectron holography.

Since the PEH term and the ESI term are comparable to the direct term, it is clear that the electron multiple scattering with atoms is important in PEH measurements. The ESI term contribution and the electron multiple-scattering contribution are expected to greatly disturb the photoelectron hologram (the PEH term). Furthermore there is a problem in the PEH term itself because of anisotropic scattering amplitudes $f_{i}^{\mathrm{e}}(|\boldsymbol{q}|)$ of electron-atom scattering. ${ }^{19}$

\section{B. Cross section of MEXH}

In order to get the cross sections of MEXH and EXAFS from the photoionization cross section Eq. (38), we have to integrate it over the photoelectron direction $\hat{\boldsymbol{p}}$. After the integration, we have ${ }^{1}$

$$
\begin{aligned}
\sigma_{\mathrm{MEXH}}(\omega, \hat{\boldsymbol{k}}, \boldsymbol{\epsilon})= & \frac{4 \pi}{3}(2 \pi)^{4} m_{\mathrm{e}}|\boldsymbol{p} \| C(\omega)|^{2} \\
& \times\left[1-\bar{\chi}^{\mathrm{x}}(\omega, \hat{\boldsymbol{k}})-\bar{\chi}^{\mathrm{e}}(\omega, \boldsymbol{\epsilon})\right],
\end{aligned}
$$

where

$$
\bar{\chi}^{\mathrm{x}}(\omega, \hat{\boldsymbol{k}})=2 r_{\mathrm{e}} \operatorname{Re} \sum_{i \neq 0}\left[1-\left(\boldsymbol{\epsilon} \cdot \hat{\boldsymbol{\xi}}_{i}\right)^{2}\right] \frac{f_{i}^{\mathrm{x}}\left(\left|\boldsymbol{l}_{i}\right|\right)}{\left|\boldsymbol{\xi}_{i}\right|} e^{i|\boldsymbol{k}|\left|\boldsymbol{\xi}_{i}\right|} e^{i \boldsymbol{k} \cdot \boldsymbol{\xi}_{i}}
$$

and

$$
\bar{\chi}^{\mathrm{e}}(\omega, \boldsymbol{\epsilon})=\sum_{i \neq 0} \frac{\left(\boldsymbol{\epsilon} \cdot \hat{\boldsymbol{\xi}}_{i}\right)^{2}}{\left|\boldsymbol{p} \| \boldsymbol{\xi}_{i}\right|^{2}} \operatorname{Im}\left[e^{-2 i\left|\boldsymbol{p} \| \boldsymbol{\xi}_{i}\right|} f_{i}^{\mathrm{e}}(2|\boldsymbol{p}|)\right] .
$$

The MEXH cross section Eq. (43) has three contributions: the direct term (the first term), the MEXH term (the second term), and the EXAFS term (the third term).

Let us estimate the relative size of each term in the cross section. The approximate ratio is given by

$$
\text { direct term: MEXH term: EXAFS term } \approx 1: \frac{Z r_{e}}{r}: \frac{Z \alpha^{2}}{2|p|^{3} r_{\mathrm{e}} r^{2}} .
$$

Although sizes of the direct term and the MEXH term do not depend on energy, the size of the EXAFS term decreases with energy as $|\boldsymbol{p}|^{-3}$. For an energy of $5 \mathrm{keV}$ above the absorption edge, and for medium heavy atoms at $r \approx$ (a few $\AA$ ), the EXAFS term is in the order of $10 \%$ of the $\mathrm{X}$-ray hologram term. The EXAFS term is not considered to be negligibly small.

The EXAFS term has a dependence on the incident photon polarization $\boldsymbol{\epsilon}$ through the factor $\left(\boldsymbol{\epsilon} \cdot \hat{\boldsymbol{\xi}}_{i}\right)^{2}$. In MEXH, one measures the MEXH cross section Eq. (43) while changing the incident photon direction $\hat{\boldsymbol{k}}$, and the dependence of the cross section on $\hat{\boldsymbol{k}}$ contains holographic information. By changing the incident photon direction $\hat{\boldsymbol{k}}$, the polarization $\boldsymbol{\epsilon}$ of the incident photon also changes, since they are vertical to each other: $\boldsymbol{\epsilon} \cdot \hat{\boldsymbol{k}}=0$. For this reason, the EXAFS term produces another $\hat{\boldsymbol{k}}$ dependence through $\boldsymbol{\epsilon}$. If we take the polar coordinate $\hat{\boldsymbol{k}}=(\sin \theta \cos \phi, \sin \theta \sin \phi, \cos \theta)$, the EXAFS term for a fixed energy behaves as $A_{0}+A_{1} \sin \left[\phi+\alpha_{1}(\theta)\right]$ $+A_{2} \sin \left[2 \phi+\alpha_{2}(\theta)\right]$, where $A_{0}, A_{1}$, and $A_{2}$ are constants. $\alpha_{1}(\theta)$ and $\alpha_{2}(\theta)$ are functions of $\theta$, and their functional forms for a given sample depend on the choice of the incident photon polarization $\boldsymbol{\epsilon}$. If we take the polarization vector $\boldsymbol{\epsilon}$ parallel to the $\theta$-rotation axis, then $\alpha_{1}$ and $\alpha_{2}$ become constants without dependence on $\theta$. With any choice of polarization vector $\boldsymbol{\epsilon}$, contributions of the EXAFS term other than constant are sinusoidal functions of $\phi$ with periodicity one and two for given energy and $\theta$. It is easy to eliminate them from the MEXH signal.

The MEXH hologram function Eq. (44) is consistent with the calculation in classical electrodynamics. ${ }^{20,21}$ The MEXH hologram function Eq. (44) may be interpreted as that Thomson scattered virtual photons distribute as $[1-(\boldsymbol{\epsilon}$ $\left.\left.\cdot \hat{\boldsymbol{k}}^{\prime}\right)^{2}\right]$, where $\boldsymbol{k}^{\prime}$ is the momentum of the virtual photon. This distribution is equivalent to that of the Thomson scattered real photons

$$
\sum_{\alpha^{\prime}} \frac{d \sigma^{\text {(Thomson) }}}{d \Omega}=\sum_{\alpha^{\prime}} r_{\mathrm{e}}^{2}\left(\boldsymbol{\epsilon}^{(\alpha)} \cdot \boldsymbol{\epsilon}^{\prime\left(\alpha^{\prime}\right)}\right)^{2}=r_{\mathrm{e}}^{2}\left[1-\left(\boldsymbol{\epsilon}^{(\alpha)} \cdot \hat{\boldsymbol{k}}^{\prime}\right)^{2}\right],
$$

where $\boldsymbol{\epsilon}^{(\alpha)}$ and $\boldsymbol{\epsilon}^{\prime\left(\alpha^{\prime}\right)}$ are polarization vectors of photons before and after Thomson scattering, respectively, and $\alpha$ and 


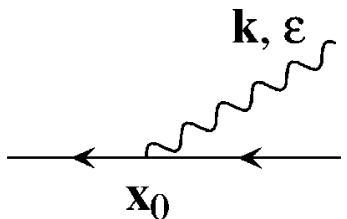

FIG. 5. The leading order contribution of the fluorescence process. The hole in a deeper bound state in the atom at $\boldsymbol{X}_{0}$ decays to the hole state in a shallower bound state by emitting the fluorescence photon.

$\alpha^{\prime}$ stand for two independent polarization states. Thus the effect of the incident photon polarization in MEXH is equivalent to the case where one regards the Thomson scattered virtual photon as the real photon. The effect of the incident photon polarization has been investigated in detail in Ref. 22.

\section{Cross section of EXAFS}

The EXAFS cross section is given by the MEXH cross section Eq. (43) with fixed incident photon direction $\hat{\boldsymbol{k}}$ and polarization $\boldsymbol{\epsilon}$, as we have seen in Eq. (6). To the EXAFS cross section there are contributions not only of the direct and the EXAFS term but also of the MEXH term. Both the EXAFS term and the MEXH term oscillate with energy. If the size of the MEXH term is not negligible, it is harmful to EXAFS experiments. The ratio of each term in the cross section is given by Eq. (46). As mentioned before, sizes of the direct term and the MEXH term are constant in energy. On the other hand, the size of the EXAFS term decreases with energy as $|\boldsymbol{p}|^{-3}$. We give ratios of the MEXH term to the EXAFS term for an atom at $r \approx$ (a few $\AA$ ). For energy $0.1 \mathrm{keV}$ above the absorption edge, the ratio is in the order of $1 \%$; and for $1.0 \mathrm{keV}$ above the absorption edge, it is in the order of 1 . Thus the effect of the MEXH term is not negligible for higher energies and can disturb the EXAFS signal.

Note that these values of the ratio are for one neighboring atom in spatially ordered samples. For samples such as powders, the MEXH term becomes smaller after averaging over contributions of photoelectron emitter atoms in a sample.

For crystalline samples near a Bragg angle, the MEXH term becomes extremely large, since a large number of scatterer atoms contribute constructively in the summation over $i$ in Eq. (44), and a strong X-ray standing wave field is formed inside a sample. ${ }^{20,23,24}$

\section{TRANSITION PROBABILITY OF XFH}

The transition probability of XFH is calculated in a similar way as the photoionization cross section. We take into account the leading order fluorescence process Fig. 5 and the fluorescence photon interaction with neighboring atoms Fig. 6 . In the interaction of the fluorescence photon with atoms, we assume the dominance of the Thomson scattering, although it is true that contributions other than the Thomson scattering become more important in XFH as compared to the case in $\mathrm{MEXH}$, since the fluorescence photon energy is always lower than the incident photon energy. The transition probability of XFH $w_{\mathrm{XFH}}$ is connected with the transition matrix $T_{f i}^{\prime}$ as follows:

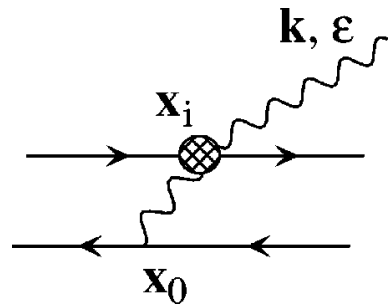

FIG. 6. The scattering of the fluorescence photon. The fluorescence photon is scattered by an electron of the atom at $\boldsymbol{X}_{i}$. In QED, the virtual photon travels in both directions. The circle with hatch represents all photon-electron interaction diagrams as Fig. 3.

$$
\frac{d w_{\mathrm{XFH}}}{d \Omega_{k}}=2 \pi \omega^{2}\left|T_{f i}^{\prime}\right|^{2},
$$

where $\omega$ and $\Omega_{k}$ are the energy and solid angle of the fluorescence photon, respectively.

The leading order fluorescence contribution, corresponding to Fig. 5, is given by

$$
T_{f i}^{\prime(0)}=\boldsymbol{\epsilon} \cdot \boldsymbol{D}(\omega) e^{-i \boldsymbol{k} \cdot \boldsymbol{X}_{0}},
$$

where

$$
\boldsymbol{D}(\omega)=\frac{e}{\sqrt{(2 \pi)^{3} 2 \omega}} \int d^{3} s_{0} \boldsymbol{J}^{\prime F I}\left(\boldsymbol{s}_{0}\right) e^{-i \boldsymbol{k} \cdot s_{0}},
$$

and $\boldsymbol{J}^{\prime F I}\left(\boldsymbol{s}_{0}\right)$ is the electromagnetic current of the fluorescence process. $\boldsymbol{k}$ and $\boldsymbol{\epsilon}$ are the momentum and polarization vector of the fluorescence photon, respectively. $\boldsymbol{X}_{0}$ is the position of the fluorescence atom.

The contribution of fluorescence photon interaction, corresponding to Fig. 6, is calculated in a similar way as in Sec. III C, and is given by

$$
\begin{aligned}
T_{f i}^{\prime(1)}= & -\frac{e^{3}}{m_{\mathrm{e}}} \sum_{m, n=1}^{3} \sum_{i} \int d^{3} r_{1} \int d^{3} r_{2} \\
& \times \frac{\epsilon_{m}}{\sqrt{(2 \pi)^{3} 2 \omega}} \rho_{i}^{\mathrm{e}}\left(\boldsymbol{r}_{1}-\boldsymbol{X}_{i}\right) \\
& \times e^{-i \boldsymbol{k} \cdot \boldsymbol{r}_{1}} G_{m n}\left(\omega, \boldsymbol{r}_{1}-\boldsymbol{r}_{2}\right) \boldsymbol{J}_{n}^{\prime F I}\left(\boldsymbol{r}_{2}-\boldsymbol{X}_{0}\right) .
\end{aligned}
$$

After making the same approximation to the photon propagator $G_{m n}\left(\omega, \boldsymbol{r}_{1}-\boldsymbol{r}_{2}\right)$ as Eq. (24), we perform integrations over $\boldsymbol{r}_{1}$ and $\boldsymbol{r}_{2}$ in Eq. (51), and obtain the result

$$
\begin{aligned}
T_{f i}^{\prime(1)}= & -r_{\mathrm{e}} e^{-i \boldsymbol{k} \cdot X_{0}} \sum_{i \neq 0}\left[\boldsymbol{\epsilon} \cdot \boldsymbol{D}(\omega)-\boldsymbol{\epsilon} \cdot \hat{\boldsymbol{\xi}}_{i} \hat{\boldsymbol{\xi}}_{i} \cdot \boldsymbol{D}(\omega)\right] \\
& \times \frac{f_{i}^{\mathrm{x}}\left(\left|\boldsymbol{l}_{i}^{\prime}\right|\right)}{\left|\boldsymbol{\xi}_{i}\right|} e^{i|k|\left|\boldsymbol{\xi}_{i}\right|} e^{-i \boldsymbol{k} \cdot \boldsymbol{\xi}_{i}}
\end{aligned}
$$

where $\boldsymbol{l}_{i}^{\prime}=\boldsymbol{k}-|\boldsymbol{k}| \hat{\boldsymbol{\xi}}_{i}$ is the momentum transfer at the Thomson scattering.

By substituting contributions of the transition matrix, Eqs. (49) and (52), to the definition of the transition probability Eq. (48), we have 


$$
\begin{aligned}
\frac{d w_{\mathrm{XFH}}(\hat{\boldsymbol{k}}, \boldsymbol{\epsilon})}{d \Omega_{k}}= & 2 \pi \omega^{2}\left[[\boldsymbol{\epsilon} \cdot \boldsymbol{D}(\omega)]^{2}-2 r_{\mathrm{e}}\right. \\
& \times \operatorname{Re} \sum_{i \neq 0}\left\{[\boldsymbol{\epsilon} \cdot \boldsymbol{D}(\omega)]^{2}-\boldsymbol{\epsilon} \cdot \boldsymbol{D}(\omega) \boldsymbol{\epsilon} \cdot \hat{\boldsymbol{\xi}}_{i} \hat{\boldsymbol{\xi}}_{i} \cdot \boldsymbol{D}(\omega)\right\} \\
& \left.\times \frac{f_{i}^{\mathrm{x}}\left(\left|\boldsymbol{l}_{i}^{\prime}\right|\right)}{\left|\boldsymbol{\xi}_{i}\right|} e^{i|\boldsymbol{k}|\left|\boldsymbol{\xi}_{i}\right|} e^{-i \boldsymbol{k} \cdot \boldsymbol{\xi}_{i}}\right] .
\end{aligned}
$$

We assume that the fluorescence emitter atoms in the sample do not have a preferred orientation as an ensemble average. With this assumption, we average the transition probability Eq. (53) over direction of $\boldsymbol{D}(\omega)$. We also sum the transition probability Eq. (53) over the polarization $\boldsymbol{\epsilon}$ of the outgoing photon, since in XFH one does not observe the polarization of the fluorescence photon. After these operations, we get the result of the transition probability of XFH:

$$
\frac{d w_{\mathrm{XFH}}(\hat{\boldsymbol{k}})}{d \Omega_{\boldsymbol{k}}}=\frac{4 \pi \omega^{2}|\boldsymbol{D}(\omega)|^{2}}{3}\left[1-\chi^{\prime \mathrm{x}}(\omega, \hat{\boldsymbol{k}})\right],
$$

where

$$
\chi^{\prime} \mathrm{x}(\omega, \hat{\boldsymbol{k}})=r_{\mathrm{e}} \operatorname{Re} \sum_{i \neq 0}\left[1+\left(\hat{\boldsymbol{k}} \cdot \hat{\boldsymbol{\xi}}_{i}\right)^{2}\right] \frac{f_{i}^{\mathrm{x}}\left(\left|\boldsymbol{l}_{i}^{\prime}\right|\right)}{\left|\boldsymbol{\xi}_{i}\right|} e^{i|\boldsymbol{k}|\left|\boldsymbol{\xi}_{i}\right|} e^{-i \boldsymbol{k} \cdot \boldsymbol{\xi}_{i}}
$$

The obtained hologram function $\chi^{\prime x}(\omega, \hat{\boldsymbol{k}})$, Eq. (55), is consistent with the calculation in classical electrodynamics. ${ }^{20}$ The factor $\left[1+\left(\hat{\boldsymbol{k}} \cdot \hat{\boldsymbol{\xi}}_{i}\right)^{2}\right]$ in Eq. (55) is identical with what is expected from the Thomson scattering of the real photon:

$$
\begin{aligned}
\frac{1}{2} \sum_{\alpha, \alpha^{\prime}} \frac{d \sigma^{(\text {Thomson })}}{d \Omega} & =\frac{1}{2} \sum_{\alpha, \alpha^{\prime}} r_{\mathrm{e}}^{2}\left(\boldsymbol{\epsilon}^{(\alpha)} \cdot \boldsymbol{\epsilon}^{\prime\left(\alpha^{\prime}\right)}\right)^{2} \\
& =\frac{r_{\mathrm{e}}^{2}}{2}\left[1+\left(\hat{\boldsymbol{k}} \cdot \hat{\boldsymbol{k}}^{\prime}\right)^{2}\right] .
\end{aligned}
$$

Finally we point out that, unlike the statement in Ref. 8, exactly the same types of Feynman diagrams contribute in both MEXH and XFH, as long as $\mathrm{x}$ rays are injected and fluorescence $\mathrm{x}$ rays are measured. The Feynman rule says that one should consider all the possible topologically distinct diagrams up to a desired order for given initial and final states. It is not possible to judge from which Feynman diagram the detected photon comes. This is a basic consideration of the uncertainty principle. Although the same Feynman diagrams contribute in MEXH and XFH, by fixing the outgoing fluorescence direction in MEXH or the incident $\mathrm{x}$ ray in $\mathrm{XFH}$, one gets a hologram in each method.

\section{SUMMARY}

We have studied MEXH, PEH, and EXAFS consistently in QED. We have argued that in order to explain these three experimental methods from their underlying photoionization process, one should take into account both the initial-state photon interaction and the final-state photoelectron interaction with neighboring atoms.

We have defined the cross sections of MEXH, PEH, and EXAFS in terms of the photoionization cross section. From the definition, it was found that the EXAFS cross section is seen as the angular integral of the PEH cross section; or it is seen as the MEXH cross section with fixed incident photon direction and polarization. We have calculated the photoionization cross section in QED including the contributions of the leading order photoionization process, the initial-state photon interaction, and the final-state photoelectron interaction. From the photoionization cross section, we have derived the cross sections of MEXH, PEH, and EXAFS. In all these cross sections, there are contributions both of the initial-state interaction and of the final-state interaction. In $\mathrm{PEH}$, the contribution of the initial-state photon interaction was found to be negligibly small typically by factor $10^{-4}$ compared to the PEH signal. The electron self-interference and the electron multiple scattering are found to be important in PEH. In MEXH, it was found that the contribution of the final-state photoelectron interaction is not negligible compared to the MEXH signal. The contribution of the final-state photoelectron interaction, however, has a simple functional form, and it is easy to eliminate from experimental data. In EXAFS, it was found that the contribution of the initial-state photon interference oscillates with energy together with the EXAFS signal. The contribution of the initial-state photon interaction is non-negligible at higher energies, and at $1 \mathrm{keV}$ above the absorption edge it can be in the same order of the EXAFS signal.

In order to make a comparison with the MEXH cross section, we have calculated the XFH transition probability. The obtained hologram functions of MEXH and XFH are found to be consistent with the calculations in classical electrodynamics.

\section{ACKNOWLEDGMENTS}

The authors thank Dr. B. Adams for discussion about comparison of hologram functions in QED with those in classical electrodynamics, and Dr. L. Tröger and Dr. P. Korecki for discussion about EXAFS.
* On leave from Japan Synchrotron Radiation Research Institute (JASRI), 1-1-1 Kouta, Mikazuki-cho, Sayo-gun, Hyogo 6795198, Japan.

${ }^{1}$ P.A. Lee, P.H. Citrin, P. Eisenberger, and B.M. Kincaid, Rev. Mod. Phys. 53, 769 (1981), and references therein.

${ }^{2}$ A. Szöke, in Short Wavelength Coherent Radiation: Generation and Applications, edited by T. Attwood and J. Boker, AIP Conf. Proc. No 147 (AIP, New York, 1986), p. 361.

${ }^{3}$ J.J. Barton, Phys. Rev. Lett. 61, 1356 (1988).
${ }^{4}$ D.K. Saldin and P.L. de Andres, Phys. Rev. Lett. 64, 1270 (1990).

${ }^{5}$ G.R. Harp, D.K. Saldin, and B.P. Tonner, Phys. Rev. Lett. 65, 1012 (1990).

${ }^{6}$ G.R. Harp, D.K. Saldin, and B.P. Tonner, Phys. Rev. B 42, 9199 (1990).

${ }^{7}$ M. Tegze and G. Faigel, Nature (London) 380, 49 (1996).

${ }^{8}$ G.A. Miller and L.B. Sorensen, Phys. Rev. B 56, 2399 (1997).

${ }^{9}$ S.Y. Tong, Hua Li, and H. Huang, Phys. Rev. Lett. 67, 3102 
(1991).

${ }^{10}$ J.J. Barton, Phys. Rev. Lett. 67, 3106 (1991).

${ }^{11}$ T. Gog, P.M. Len, G. Materlik, D. Bahr, C.S. Fadley, and C. Sanchez-Hanke, Phys. Rev. Lett. 76, 3132 (1996).

${ }^{12}$ S.Y. Tong and H. Huang, Surf. Rev. Lett. 5, 971 (1998).

${ }^{13}$ P. Korecki, J. Korecki, and T. Ślȩzak, Phys. Rev. Lett. 79, 3518 (1997).

${ }^{14}$ L. Fonda, Phys. Status Solidi B 201, 329 (1997).

${ }^{15}$ V. B. Berestetskii, E. M. Lifshitz, and L. P. Pitaevskii, in Quantum Electrodynamics, edited by L. D. Landau and E. M. Lifshitz, Vol. 4 of Course of Theoretical Physics, 2nd ed. (Pergaman, Oxford, 1982).

${ }^{16}$ J. J. Sakurai, in Modern Quantum Mechanics, edited by S. F. Tuan (Benjamin/Cummings, Menlo Park, 1985).

${ }^{17}$ In Eq. (10) of Ref. 8 the photon propagator in the Feynman gauge is used; on the other hand in Eq. (19) of Ref. 8 the threedimensional transverse gauge is applied. In QED one has to use a unique gauge fixing condition throughout calculations. Be- cause of the inconsistent choices of gauge fixing conditions, Eq. (10), Eq. (20), and the following equations in Ref. 8 should be modified by using a correct photon propagator in the threedimensional transverse gauge.

${ }^{18}$ International Tables for Crystallography, edited by A. J. C. Wilson (Kluwer Academic, Dordrecht, 1992), Vol. C.

${ }^{19}$ D.K. Saldin, Surf. Rev. Lett. 4, 441 (1997), and references therein.

${ }^{20}$ B. Adams, D.V. Novikov, T. Hiort, G. Materlik, and E. Kossel, Phys. Rev. B 57, 7526 (1998).

${ }^{21}$ In equations for MEXH (reciprocal holography), Eqs. (15) and (16) in Ref. 20, the phase factor should read $e^{i\left(k\left|\boldsymbol{r}_{j}\right|+\boldsymbol{k} \cdot \boldsymbol{r}_{j}\right)}$ because of the different wave direction in XFH and MEXH.

${ }^{22}$ P.M. Len, T. Gog, D. Novikov, R.A. Eisenhower, G. Materlik, and C.S. Fadley, Phys. Rev. B 56, 1529 (1997).

${ }^{23}$ B. W. Batterman and H. Cole, Rev. Mod. Phys. 36, 681 (1964).

${ }^{24}$ T. Gog, D. Bahr, and G. Materlik, Phys. Rev. B 51, 6761 (1995). 